\title{
Reversal training facilitates acquisition of new learning in a Morris water maze
}

\author{
José A. Alcalá ${ }^{1}$ • José E. Callejas-Aguilera ${ }^{1}$ • James Byron Nelson ${ }^{2}$ • Juan M. Rosas ${ }^{1}$
}

Published online: 20 August 2019

(C) The Psychonomic Society, Inc. 2019

\begin{abstract}
Two experiments determined the effect of interference training on subsequent spatial learning in a Morris water maze. Rats first learned that a platform was located in a quadrant marked by landmarks A and B. Different groups of rats either continued or reversed that training. In the reversal condition the platform was opposite to the initially trained quadrant. On test, a new cue, $\mathrm{C}$, was added and the platform was located in the new AC quadrant. Rats that had received the reversal training learned the location of the new platform faster than rats trained with the same platform throughout. In Experiment 2, phase 1 training was conducted by placing the rats on the platforms to ensure that they were located. Experimental rats received a reversal of the platform position in phase 2. A control group received training with both platforms present, and thus had experience with each. When the platform was then located in the new AC quadrant the rats that received reversal training learned the new location faster than those without reversal training. Results are discussed in terms of the effect of interference on the arousal of general attention.
\end{abstract}

Keywords Attention $\cdot$ Facilitation of learning $\cdot$ Interference $\cdot$ Spatial learning

\section{Introduction}

Recent associative explanations of detrimental context-switch effects on performance assume that sudden increases in prediction error, such as the ones that occur in extinction or in a discrimination reversal, lead organisms to focus their attention on the context (e.g., Bouton, 1993). Such attention is assumed to make retrieval of information learned in that context, contextspecific, regardless of whether it is the information that causes the prediction error (Bouton, 1997), or any information learned within an attended context (Rosas, Callejas-Aguilera, RamosÁlvarez, \& Abad, 2006).

The idea of prediction error modulating attention is far from new in learning theory. Attentional models of associative learning have assumed that animals pay attention to either those stimuli for which prediction error is low (Mackintosh, 1975), or to those for which prediction error is high (Pearce \&

José E. Callejas-Aguilera jecalle@ujaen.es

1 Department of Psychology, Universidad de Jaén, Jaén, Spain

2 Department of Basic psychological processes and their development, University of the Basque Country (UPV/EHU), Donostia-San Sebastián, Spain
Hall, 1980). Le Pelley (2004; see also Le Pelley, Mitchell, Beesley, George, \& Wills, 2016), integrated both ideas suggesting that in situations in which the relationship between cues is stable over time the organism engages an "attentional exploitation" mechanism, paying more attention to the better predictors in order to get maximum benefits from what was learned (Le Pelley, Beesley, \& Griffiths, 2011). However, in situations with high levels of uncertainty, the organism is assumed to engage an "attentional exploration" mechanism, spending more time processing cues that are uncertain predictors of the outcomes (Beesley, Nguyen, Pearson, \& Le Pelley, 2015). This idea of paying more attention to uncertain or novel situations was already suggested by Larrauri and Schmajuk (2008), assuming that animals respond to novelty by increasing attention to the environment. Novelty is expressed as a function of the overall prediction error in the environment; the difference between the total environmental representation expected and that which occurs. Novelty increments lead to increases in the organisms' attention, enhancing associations among the elements present in the situation. As learning progresses and the environment is better represented, attention is assumed to progressively decrease (see also Schmajuk, Lam, \& Gray, 1996).

All these models have a common assumption that an increase in prediction error will impact learning. Some reports in the literature suggest that this impact may be seen as the 
facilitation of subsequent learning (e.g., Easdale, Le Pelley, \& Beesley, 2019; Hall \& Pearce, 1982; Liberman, 1951; Shanab \& Cotton, 1970; see also Courville, Daw, \& Touretzky, 2006). For instance, a recent study from our laboratory has found that rats acquired temporal conditioning faster when it was preceded by a discrimination reversal than when it was preceded by simple discrimination (Alcalá, Callejas-Aguilera, Lamoureux, \& Rosas, 2019). In an appetitive conditioning procedure rats received $\mathrm{CS} 1+/ \mathrm{CS} 2-$ training in acquisition, followed either by more acquisition or by reversal (CS1-/CS2+) training. Afterwards, food was delivered every 60 (Experiments 1, 2, and 3) or 30 (Experiment 3) s. Rats showed temporal conditioning in the form of greater responding prior to the delivery of food relative to after its delivery, and that discrimination along time was acquired more rapidly in the reversal group than in both the group that only had the discrimination experience and the naïve control without discrimination experience (Experiment 2). Presumably, the reversal produced interference and novelty, increasing attention to the elements of the situation. A similar result has recently also been demonstrated in humans where simple extinction of a CS facilitated subsequent learning about regularly timed, but otherwise un-signaled, events (Nelson, Fabiano, \& Lamoureux, 2018). In a related result, González, Alcalá, Callejas-Aguilera, and Rosas (2019) found that experiencing extinction with an unrelated cue facilitated reversal of a conditioned inhibitor in human predictive learning. However, the effect of the interference experience upon new learning is not necessarily ubiquitous. For instance, Alcalá, González, Aristizabal, Callejas-Aguilera, and Rosas (2018) found that the experience of a discrimination reversal facilitated context conditioning, but it did not facilitate learning about a CS-US new relationship learned concurrently with the reversal experience. These conflicting results prompt the need for further research that will help to understand the generality and the limitations of facilitation of learning by interference experiences.

The goal of the research reported here was to further explore the effects of interference on new learning using spatial learning in rats. Despite some controversy in the literature, there seems to be little doubt today that spatial learning involves many of the same principles as other forms of associative learning (e.g., Chamizo, 2003, Pearce, 2009; but see Jeffery, 2010). Basic associative learning phenomena have been found in spatial learning such as latent inhibition (Prados, Chamizo, \& Mackintosh, 1999), spontaneous recovery (Prados, Manteiga, \& Sansa, 2003), partial reinforcement (Prados, Sansa, \& Artigas, 2008), blocking (Roberts \& Pearce, 1999), and renewal (Lattal, Mullen, \& Abel, 2003). Thus, it was expected that the experience of interference would have similar effects in aversively motivated spatial learning to those reported in rats' Pavlovian (e.g. Alcalá et al., 2019) and operant appetitive (Liberman, 1951; Shanab \& Cotton, 1970) conditioning and in human learning (Easdale et al., 2019; González et al., 2019; Nelson et al., 2018), rather than the null effect of interference upon concurrent acquisition of simple conditioning reported by Alcalá et al. (2018). Two experiments were conducted in a Morris water maze in which different landmarks signaled the position of a hidden platform. The goal was to determine whether an interference experience in the water maze would facilitate subsequent learning about a new location of the hidden platform compared to a group of rats that did not have the interference experience.

\section{Experiment 1}

Experiment 1 determined whether training animals in a water maze with the platform sequentially hidden in two different positions facilitates learning a platform location in a new position compared to animals for which the location of the platform was consistent during training. The design of the Experiment is presented in the top section of Fig. 1. The experiment included three groups of rats. Rats in groups Reversal (R) and Acquisition-long (AL) were first trained with the platform placed between two distinctive landmarks (A and $\mathrm{B}$ ) while rats in group Acquisition-short (AS) were kept in their home cages. During phase 2, rats in group AS began the phase-1 acquisition treatment while rats in group AL continued that training. Group R received the platform hidden in Q3, the quadrant opposite from its phase 1 position (see Lattal et al., 2003). Finally, all rats entered phase 3, in which a new landmark was added (C) and the platform was hidden in the new $\mathrm{AC}$ quadrant, equidistant from the prior platform placement for all the groups.

As discussed earlier, Alcalá et al. (2018) found no facilitation with a simple novel CS in Pavlovian conditioning following reversal training. We assumed that lack of effect to be due to a limit to which the reversal can increase attention to novel stimuli that already command substantial attention (Larrauri \& Schmajuk, 2008). The design of phase 3 was intended to limit the effect of novelty. To find the platform, the animals must attend to the presence of two cues which will be comprised of a novel, and at least one familiar landmark, the latter of which is already associated with a different platform position. We expected that reversal training should enhance learning about these combinations of novel and familiar stimuli, at least partially reducing the impact of initially high attention to only the novel landmark.

The length of training in group AL could have strengthened the habit to choose the original position of the platform, leading to a retardation in the acquisition of new learning that could be wrongly interpreted as facilitation of learning in group R. Group AS controlled for that possibility, as it received the same length of training with the original position of the platform as group R. All animals had sufficient experience with the task and apparatus immediately before phase 3 , thus differences during the critical phase 3 should be a product 


\section{Experiment 1}

\begin{tabular}{|c|c|c|c|}
\hline & Phase 1 & Phase 2 & Phase 3 \\
\hline Reversal (R) & B & B & \\
\hline Acquisition-Long (AL) & B & & \\
\hline Acquisition-Short (AS) & - & B & \\
\hline
\end{tabular}

\section{Experiment 2}

\begin{tabular}{|c|c|c|c|}
\hline & Phase 1 & Phase 2 & Phase 3 \\
\hline Reversal (R) & & & \\
\hline Acquisition (A) & & & \\
\hline
\end{tabular}

Fig. 1 Experimental designs of Experiment 1 (top panel) and Experiment 2 (bottom panel). Letters represent the landmarks. A was square or circle counterbalanced; B and C were fixed or flashing lights counterbalanced. Q1-Q4 identifies the quadrants used throughout

of differences in the learning experience (Acquisition vs. Reversal), and not because of different degrees of experience with the learning environment immediately prior to testing. If interference produces a general increase in attention, then the reversal experience should facilitate acquisition of new learning. Group $\mathrm{R}$ should associate the landmarks with the new platform location more quickly than the other two groups.

\section{Method}

\section{Subjects}

The present experiments were approved by the bioethical committee of the Universidad de Jaén, reference JA-U1 2013. The subjects were 24 female Wistar rats, approximately 6 months old at the beginning of the experiment. The rats had experience in a classical conditioning experiment, unrelated to this one, in which they had limited access to food. They were kept for 3 weeks with food and water available ad libitum before starting this experiment. Animals were individually housed in standard cages in a room with a 12:12 h light-dark cycle, and were maintained ad libitum throughout the experiment. Experimental sessions were conducted in the first $6 \mathrm{~h}$ of the light cycle (9:00-14:00). Rats were randomly assigned to groups AL, AS, and R before starting the experiment. One of the rats in group AL was removed from the experiment as she showed behavioral symptoms of stress and somehow injured herself in the home cage. Group AL included seven animals, while AS and R groups consisted of eight each.

\section{Apparatus}

The experiment was conducted in a circular water maze tank similar to the one used by Morris (1981). The tank measured 
$1.40 \mathrm{~m}$ in diameter and $0.6 \mathrm{~m}$ deep. It was filled to a depth of $0.48 \mathrm{~m}$ with water rendered opaque by the addition of black watercolor paint. The water temperature was maintained at 22 $\pm 1{ }^{\circ} \mathrm{C}$. The pool was placed in the middle of a square room and mounted on a metal platform $0.27 \mathrm{~m}$ above the floor. The pool was surrounded by opaque curtains from the edge of the pool to the ceiling. A video camera was mounted $1.8 \mathrm{~m}$ above the center of the pool and was controlled by a computer in the adjacent room to record the rats' activity. Swimming patterns were analyzed by Smart Video Tracking Software 2.0 developed by Panlab, Harvard Apparatus S.A. (Cornellá, Barcelona, Spain). A circular $0.47-\mathrm{m}$ high platform $(10 \mathrm{~cm}$ diameter) was used as a hidden escape platform. Once placed in the tank the platform was hidden $1 \mathrm{~cm}$ below the surface of the water

Three different landmarks were used during the experiment. Landmark A was always a geometrical shape: a thin colored polystyrene circle with $(19.5 \mathrm{~cm}$ of radius $\times 3.4 \mathrm{~cm}$ depth) or a thin black and white polystyrene square $(29 \times 29 \times$ $1 \mathrm{~cm})$ held from the ceiling. Steady or flashing $(120-\mathrm{hz})$ yellow lights were orthogonally counterbalanced as landmarks B and C. Landmarks were placed at the edge of the pool $20 \mathrm{~cm}$ above the water surface. Landmark positions, and consequently the position of the landmark-contingent platform, were rotated from trial to trial. Throughout, for further identification purposes, Q1 was the quadrant marked by the AB landmarks and quadrants Q2-Q4 were the next consecutive clockwise quadrants.

\section{Procedure}

Following the procedure used by Chamizo, Rodríguez, Espinet, and Mackintosh (2012), three types of swimming trials were used at different points along the experiment: Pretraining trials, Training trials, and Probe trials. Pretraining trials were conducted with the platform present in different quadrants, but without landmarks available. Training trials had both the platform and landmarks available. In Probe trials only landmarks, but not the platform, were available. The maximum duration of each trial where the platform was present was $90 \mathrm{~s}$. When the animal reached the platform, it was allowed to stay there for $30 \mathrm{~s}$. Rats were given $60 \mathrm{~s}$ to locate the platform on trials where it was present. Rats that did not locate the platform in $60 \mathrm{~s}$ were gently pushed toward the platform where they remained for $30 \mathrm{~s}$. On probe trials rats were allowed to swim for $60 \mathrm{~s}$, and then removed from the tank. The intertrial interval was variable, averaging $12.5 \mathrm{~min}$ (range 10$15 \mathrm{~min})$. During the ITI rats were placed into individual plexiglas boxes in a different room from where the training was conducted. The design of Experiment 1 is presented in top section of Fig. 1.
Pretraining (Days 1-2) During the pretraining phase all rats received five trials in which they were introduced into the pool with the platform hidden in a different quadrant from trial to trial, without landmarks available. Rats were introduced into the pool from a different position (North, East, South, West) on each trial throughout the experiment. Two trials were conducted on Day 1, and three on Day 2. Pretraining for group Acquisition-Short was conducted on Days 9 and 10.

Phase 1 (Days 3-10) Rats in groups AL and R received 48 training trials (six trials per day) over eight consecutive days, while rats in group AS remained in their home cages. As shown in the top section of Fig. 1, the platform was hidden in the middle of the quadrant between landmarks A and B. A seventh probe trial was added every other day of training.

Phase 2 (Days 11-18) Phase 2 was identical to phase 1 for all groups, except where noted. Group AL continued training with the landmark in the AB quadrant, and group AS began training with the landmark in the $\mathrm{AB}$ quadrant. In group $\mathrm{R}$ the position of the platform was changed from Q1 to Q3, the quadrant opposite that marked by the A and B landmarks (see Fig. 1; see also Lattal et al., 2003, Experiment 2).

Phase 3 (Days 19-24) An additional landmark (C) was introduced across from landmark B (see Fig. 1). The platform was placed in the center of Q4, the quadrant defined by landmarks $A$ and $C$, which was new and equidistant from where the platform had been hidden before for all rats. Thirty-six training trials were conducted over six consecutive days. The seventh trial of each day was a probe trial.

\section{Data analysis}

Latency to reach the platform was recorded on each trial. Because each rat was started from one of the four different axes (North, East, South, West) randomly on each trial, and each session contained six trials, we averaged across sessions in two-session blocks to better ensure that the measures of latency averaged equally across the possible starting locations.

The time that rats spent swimming in each quadrant was recorded during each probe trial. The data were arranged into halves of training (two-session blocks in phases 1 and 2, threesession blocks in phase 3 ) for clarity in presentation. All data were analyzed with Group $\times$ Quadrant $\times$ Block analyses of variance (ANOVA) including the time spent in all four quadrants. Because the length of the probe trials was fixed across probes and groups, there can be no main effects of Group or Block. What is important is how the rats divided their time in the four quadrants, and how that changed between groups over training as reflected in the Quadrant $\times$ Block, Group $\times$ Quadrant, and Group $\times$ Quadrant $\times$ Block interactions. 
Confidence intervals on partial-eta squares $(95 \%)$ were computed using software available in Nelson (2016).

\section{Results and discussion}

\section{Latency}

Phase 1 The latency data are shown in Fig. 2. A Group $\times$ Block ANOVA of phase 1 showed an effect of Block, $F(3,39)=3.17, p=.035, \eta^{2}=.20,95 \%$ confidence interval (CI) $[.000002, .36]$ and no other effects, $p \mathrm{~s} \geq .55$. Both group $\mathrm{R}$ and group $\mathrm{AL}$ decreased the time needed to locate the platform across phase 1 .

Phase 2 The same analysis of phase 2 (now including group Short) showed an effect of Block, $F(3,60)=6.78, p=.001, \eta^{2} p$ $=.25,95 \% \mathrm{CI}[.06, .39]$; no other effects or interaction were significant, $p s \geq .1$. The two groups being trained on a new location (R and AS) clearly did not differ. Contrasting those two groups combined against group $\mathrm{AL}$ did produce an effect of Group, $F(1,21)=5.39, p=.03, \eta_{p}^{2}=.20,95 \%$ CI [.000001, $.45]$. It took the groups learning a new location somewhat longer to find the platform than group $\mathrm{AL}$, which was returning to a familiar platform location, though any effect clearly was not robust.

Phase 3 A Group $\times$ Block ANOVA of phase 3 showed an effect of Block, $F(2,40)=4.21, p=.02, \eta_{p}^{2}=.17,95 \%$ CI $[.001, .35]$, and no effect of Group, $F(2,20)=1.56, p=$ .23 , and no interaction, $F(4,40)=1.02, p=.41$. Despite the trend for the reversal group to locate the platform more rapidly, there were no reliable group differences in the latency data.

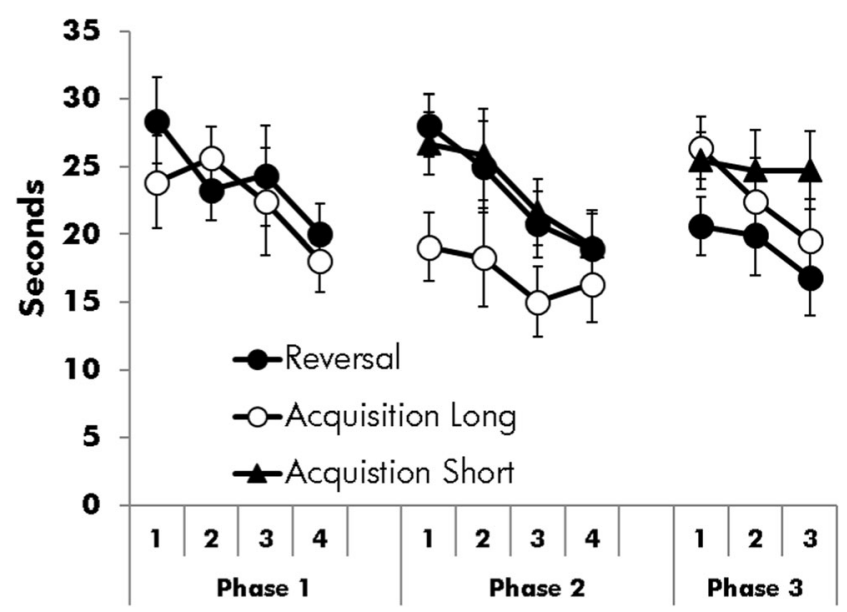

Fig. 2 Latency to reach the platform in each phase of Experiment 1, averaged across two-session blocks. Vertical lines represent the standard error of the mean

\section{Probe trials}

Phase 1 The data from the probe trials are shown in Fig. 3. The analysis revealed a Quadrant $\times$ Block interaction, $F(3,39)=$ $3.34, p=.028, \eta_{p}^{2}=.20,95 \%$ CI $[.000002, .36]$. No other effects whose interpretation is not superseded by the interaction were reliable, $p \mathrm{~s} \geq .988$.

The second block of training generally reflected the overall pattern shown on both blocks, and as the performance toward the end of the phase is of most importance we conducted follow-up analyses of the Quadrant effect on the second block. Rats swam more in $\mathrm{Q} 1$, the target $\mathrm{AB}$ quadrant, than in the other three, $F_{\mathrm{S}}(1,14) \geq 32.96, p \mathrm{~s} \leq .0001, \eta^{2} \geq .70,95 \% \mathrm{CI}$ $[.33, .82]$. Rats swam less in $\mathrm{Q} 3$, opposite the target quadrant, than in $\mathrm{Q} 2$ or $\mathrm{Q} 4, F \mathrm{~s}(1,14) \geq 6.79, p \mathrm{~s} \leq .02, \eta_{p}^{2} \geq .33,95 \% \mathrm{CI}$ $[.004, .58]$. Time spent in Q2 and Q4 did not differ, $F(1,14)=$ $3.12, p=.099$.

Phase 2 The analysis of phase 2 revealed a Group $\times$ Quadrant $\times$ Block interaction, $F(6,60)=5.83, p=.00008, \eta_{p}^{2}=.37$, $95 \% \mathrm{CI}[.12, .47]$, superseding the interpretation of any other effect. There were Group $\times$ Quadrant interactions on each block, $F \mathrm{~s}(3,60) \geq 2.69, p \mathrm{~s} \leq .02, \eta_{p}^{2} \geq .21,95 \%$ CI [.03, $.35]$. We followed those two-way interactions by examining the effects of quadrants within each block in each group.

On the first block there was an effect of Quadrant in groups $\mathrm{R}, F(3,21)=5.88, p=.008, \eta^{2}{ }_{p}=.46,95 \% \mathrm{CI}[.07, .62]$, and $\mathrm{AL}, F(3,18)=8.27, p=.001, \eta_{p}^{2}=.58,95 \%$ CI $[.16, .71]$. There was no effect of Quadrant in group AS, $F(3,21)=1.15$, $p=.35$.

Quadrant effects, Group R, phase 2, block 1 In group R rats spent less time in Q2 than in Q1 or Q4, $F \mathrm{~s}(1,7) \geq 12.65, p \mathrm{~s} \leq$ $.009, \eta_{p}^{2} \geq .64,95 \%$ CI $[.06, .80]$, and the difference between Q2 and Q4 approached reliability, $F(1,7)=5.44, p=.05, \eta^{2}{ }_{p}=$ $.44,95 \%$ CI $[.00001, .70]$. There were no other differences, $F_{\mathrm{s}}(1,7) \leq 1.9, p \mathrm{~s} \geq .21$.

Quadrant effects, Group AL, phase 2, block 1 In group AL rats continued to swim longer in $\mathrm{Q} 1$, the $\mathrm{AB}$ target quadrant, than the in other three quadrants, $F(1,7) \geq 6.02, p \mathrm{~s} \leq .0495, \eta^{2}{ }_{p}>$ $.50,95 \%$ CI $[.0001, .71]$. Time spent swimming in the other three quadrants did not differ, $F_{\mathrm{s}}(1,7) \leq 3.89, p \mathrm{~s} \geq .096$.

On the second block there were effects of quadrant in group $\mathrm{R}, F(3,21)=21.05, p<.0001, \eta_{p}^{2}=.75,95 \%$ CI $[.46, .82]$, and group $\mathrm{AL}, F(3,18)=15.79, p<.0001, \eta^{2}{ }_{p}=.72,95 \% \mathrm{CI}$ $[.37, .81]$. Unlike the first block, there was a reliable effect of quadrant in group AS on the second block, $F(3,21)=4.00, p=$ $.02, \eta_{p}^{2}=.36,95 \%$ CI $[.006, .54]$.

Quadrant effects, Group R, phase 2, block 2 In group R more time was spent in Q3, the new target area, than in any of the other three quadrants, $F_{\mathrm{s}}(1,7) \geq 24.83, p \mathrm{~s} \leq .002, \eta_{p}^{2} \geq .78$, 


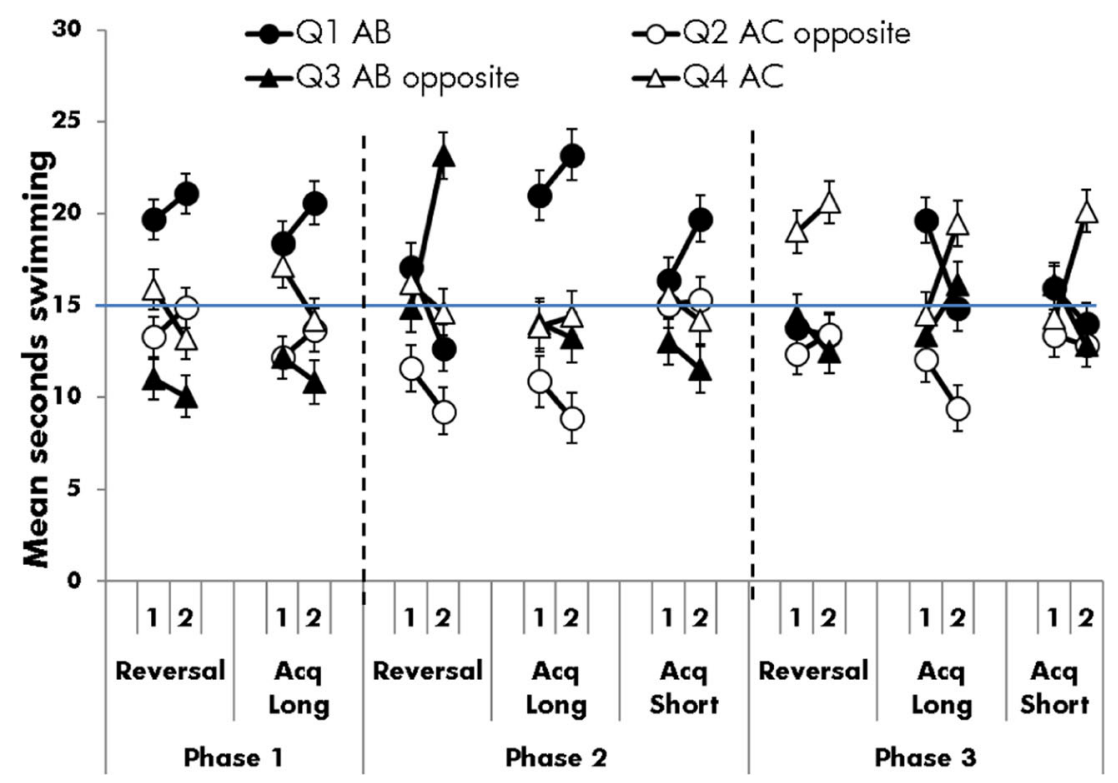

Fig. 3 Mean time spent in each quadrant during the probe trials in each half of each phase of training and testing in Experiment 1. The horizontal line at $15 \mathrm{~s}$ indicates chance performance. Vertical lines on points show

95\% CI $[.24, .88]$. Rats spent less time in Q2 than in Q1 or in $\mathrm{Q} 4, F_{\mathrm{s}}(1,7) \geq 6.47, p \mathrm{~s} \leq .038, \eta^{2}{ }_{p} \geq .48,95 \%$ CI $[.00001, .72]$. Time spent in Q1 and Q4 did not differ, $F<1$.

Quadrant effects, Group AL, phase 2, block 2 In group AL rats continued to swim in the Q1 target quadrant more than the other three, $F \mathrm{~s}(1,6) \geq 8.19, p \mathrm{~s} \leq .029, \eta_{p}^{2} \geq .57,95 \% \mathrm{CI}$ $[.00001, .78]$. They swam less in Q2 than in Q3 or Q4, $F_{\mathrm{S}}(1,6) \geq 9.16, p \mathrm{~s} \leq .02, \eta_{p}^{2} \geq .60,95 \%$ CI $[.005, .79]$. Time spent in Q3 or Q4 did not differ.

Quadrant effects, Group AS, phase 2, block 2 In group AS rats preferred the Q1 AB target quadrant over Q3, which was opposite to the target, $F(1,7)=8.77, p=.02, \eta_{p}^{2}=.56,95 \%$ CI $[.01, .76]$. However, there were no other differences involving Q1, or any other quadrants, $F \mathrm{~s}(1,7) \leq 3.74, p \mathrm{~s} \geq .094$.

By the end of phase 2, group $\mathrm{R}$ reversed its phase 1 performance and came to favor the new Q3 target area over the other areas. Group AL continued its preference for the initially trained $\mathrm{Q} 1 \mathrm{AB}$ target area, and group AS developed a mild preference for the $\mathrm{Q} 1 \mathrm{AB}$ target area by the end of its training.

If the areas are considered by their status as "target" or not in phase 2, then the performance in group Reversal and Acquisition Long is clearly identical in the second block. Both groups prefer their target area, both groups tend to avoid Q2, and there is no difference between the remaining areas. This equality in performance between the two groups, which had the same amount of experience in the pool at this point in the experiment, is important. In phase 2 it is possible that Group Reversal learned to search for new platform locations when the original platform was not encountered. The platform the standard error of the mean with between-subjects differences removed to facilitate between-quadrant comparisons within groups

was absent on the test, so if they had learned such a "lose switch" strategy, then this group should have shown a different swimming pattern at the end of training on the probe tests. With a lose-switch strategy, the reversal rats should spend less time in the target quadrant than the other groups as they should be expecting the platform in a new location once its absence is encountered. But, as shown in Fig. 3, the patterns of swimming were identical across all quadrants, making that interpretation unlikely.

Phase 3 The analysis of phase 3 revealed a Group $\times$ Quadrant $\times$ Block interaction, $F(6,60)=2.95, p=.01, \eta_{p}^{2}=.23,95 \% \mathrm{CI}$ $[.0001, .71]$, that superseded any other effect. There was a Group $\times$ Quadrant interaction in the first half of phase 3, $F(6,60)=3.64, p=.004, \eta_{p}^{2}=.27,95 \%$ CI $[.04, .37]$, but not in the second half, $F(6,60)=1.87, p=.1$. In the second half, there was only an effect of Quadrant, $F(3,60)=25.57, p$ $<.0001, \eta_{p}^{2}=.56,95 \%$ CI $[.37, .66]$.

In the first half there was an effect of Quadrant in group $\mathrm{R}$, $F(3,21)=6.6, p=.003, \eta^{2}{ }_{p}=.48,95 \%$ CI $[.10, .63]$. Rats in group R preferred the new AC target quadrant over the other three, $F \mathrm{~s}(1,7) \geq 9.74, \eta_{p}^{2} \geq .57,95 \% \mathrm{CI}[.02, .77]$. There were no differences among the other three quadrants, $F_{\mathbf{s}}(1,7) \leq$ $3.99, p \mathrm{~s} \geq .086$.

In the first half there was an effect of quadrant in group $\mathrm{AL}$, $F(3,18)=6.2, p=.004, \eta^{2}{ }_{p}=.51,95 \%$ CI $[.09, .66]$. Unlike the rats in group $\mathrm{R}$, rats in group $\mathrm{AL}$ continued to prefer the previous $\mathrm{AB}$ target quadrant over the other three, $F_{\mathrm{s}}(1,6) \geq$ $6.43, p \mathrm{~s} \leq .04, \eta_{p}^{2} \geq .52,95 \%$ CI $[.000001, .75]$. There were no differences among the other three quadrants, $F_{\mathrm{s}}(1,6) \leq$ $1.08, p \mathrm{~s} \geq .15$. 
In group AS there was no preference for any area on the first block. The effect of Quadrant was not reliable, $F<1$.

In the second block, the Quadrant effect along with the absence of a Quadrant $\times$ Group interaction suggests that all groups showed the same pattern of swimming, mostly preferring the new AC target quadrant over the others. Simple effects generally confirmed that impression, with some minor deviations in group AL.

There was a quadrant effect in both group R and group AS, $F_{\mathrm{s}}(3,21) \geq 7.7, p \mathrm{~s} \leq .001, \eta_{p}^{2} \geq .52,95 \%$ CI [.13, .66]. Both groups preferred the new AC target quadrant over the other three, $F \mathrm{~s}(1,7) \geq 7.61, p \mathrm{~s} \leq .028, \eta_{p}^{2} \geq .52,95 \%$ CI [.000001, .74]. The three non-target areas did not differ from each other, $F \mathrm{~s}(1,7) \leq 1.11, p \mathrm{~s} \geq .32$.

In group $\mathrm{AL}$ there was an effect of quadrant, $F(3,18)=9.1$, $p=.0007, \eta_{p}^{2}=.60,95 \% \mathrm{CI}[.18, .72]$. Rats swam more in the target quadrant than in the other quadrants, $F \mathrm{~s}(1,6) \geq 11.58, p \mathrm{~s}$ $\leq .01, \eta_{p}^{2} \geq .66,95 \%$ CI $[.04, .82]$, with the exception of Q3, for which the difference was not reliable, $F(1,6)=3.18, p=$ .12. Rats swam less in $\mathrm{Q} 2$, the quadrant opposite the $\mathrm{AC}$ target, than in the other areas, $F_{\mathrm{S}}(1,6) \geq 6.26, p \mathrm{~s} \leq .04, \eta_{p}^{2} \geq$ $.51,95 \%$ CI $[.000001, .74]$.

Rats in all groups successfully learned to find a platform located between two landmarks in phase 1, and showed a preference for that quadrant in swim tests with the platform absent. In the second phase, the location of the platform was moved to the quadrant opposite the phase 1 target quadrant in group R, and was maintained in its original position in group AL. Group AS began training at this time with the platform in the $\mathrm{AB}$ quadrant. By the end of phase 2, rats in group $\mathrm{R}$ showed a clear preference for the new target quadrant in swim tests, while both groups AL and AS preferred their original AB quadrant.

In the final phase, all rats received the platform in a new quadrant that was equidistant to the phase 1 and 2 target quadrants. Rats in the group $\mathrm{R}$ developed a preference for swimming in the new quadrant faster than either of the other two groups, with the preference evident in the first half of training on probe trials. Rats in group AL developed a weak preference for the new area. They appeared to quickly learn where the platform was not located, but persisted somewhat in visiting the location where the platform was originally located in the first half of training. Rats in group AS did not show group AL's initial tendency to prefer the original platform, and came to prefer the new location over all others by the final session. Although the groups did not differ in their latency during phase 3 , there was a trend suggesting that group $\mathrm{R}$ could find the platform faster than both A groups. This pattern complements the overall results observed during probe trials. Latency to reach the platform on training trials appears to be less sensitive than time spent swimming in a target quadrant on probe trials (see also, e.g., Chamizo et al., 2012). That differential sensitivity is likely why probe trials are more commonly reported than is latency in these types of procedures (e.g., Lattal et al., 2003).

The results suggest that interference in phase 2 facilitated associating the platform with the new landmark cues. The results are not likely to be explained by the rats in group $\mathrm{R}$ simply being reinforced for the extra platform searching in the reversal phase. If that had occurred, then when no platform was present during the swim test the rats should have been biased to quickly abandon searching in an area where the platform was no longer located in favor of searching new areas. However, the data clearly show that the rats in this group preferred searching in the area where the platform had been most recently located. Rather than adopting a strategy, it appears as if rats in group $\mathrm{R}$ simply associated the platform with the new location cues more rapidly than the other groups.

Another possibility arises from the fact that the interference treatment is confounded with the distance of the platform from the cues. Immediately prior to phase 3, rats in group $\mathrm{R}$ had received training where the platform was far from the cues, while the other groups were trained with the platform close to the cues. If anything is learned, the rats in group $\mathrm{R}$ should learn to search far from cues, while those in the other groups should learn to search near the cues. On the swim test in phase 3 , when the platform in absent, searching in group $\mathrm{R}$ should be directed toward the quadrant most distant from all cues, which would be the "Q2 AC Opposite" quadrant shown in phase 3 of Fig. 3. As can be seen in the figure, there was no such trend in the Reversal group.

Nevertheless, there could be other effects related to having the platform hidden in different areas that are not easily anticipated and are unrelated to interference. Experiment 2 was designed to determine the effect of interference on new spatial learning in a situation in which every animal had the opportunity to learn that the platform could be hidden in different places with respect to the cues, but only group $\mathrm{R}$ received an interference treatment. Therefore, only in group $\mathrm{R}$ should attention be raised and facilitate new learning.

\section{Experiment 2}

The goal of Experiment 2 was to evaluate whether prior interference facilitates new learning while controlling for the differential experience with the position of the platform across groups. The design of Experiment 2 is presented in the bottom section of Fig. 1. Group Acquisition (A) was trained with two different platforms simultaneously placed close to, or far from, the landmarks. These are the positions of the platform that group $\mathrm{R}$, from the previous experiment, experienced across phases 1 and 2. To ensure that rats had experienced the two positions of the platform, the rats were directly placed in the platform with the landmarks present. The same procedure was applied to group Reversal (R) with the exception that only one 
platform was used. This type of latent learning has been shown to be effective in learning the location of a platform (Horne, Gilroy, Cuell, \& Pearce, 2012; Sutherland \& Linggard, 1982), although we are unaware of whether it has been conducted with two platforms.

During phase 2 both groups were required to swim and find the platforms. In group R the platform was placed in the opposite quadrant to the one where it was placed before, whereas rats in group A had both platforms available. Therefore, both groups had the opportunity to learn that the platform could be close to or far from the landmarks and, by the end of training, both groups had experienced the ambiguity of the platform being placed in two different positions of the pool, but only group $\mathrm{R}$ received the interference training, which should be the critical factor (see Nelson \& Callejas-Aguilera, 2007). Phase 3 was identical to the one conducted in the previous experiment with the platform placed in a new position between cue $\mathrm{A}$ and the added cue $\mathrm{C}$.

\section{Method}

\section{Subjects}

Sixteen female Wistar Rats, approximately 6 months old at the beginning of the experiment, were used. As in Experiment 1, rats had prior experience in a classical conditioning experiment, unrelated to this one, in which they had limited access to food. They were maintained in the same conditions as in Experiment 1.

\section{Apparatus}

The apparatus was identical to Experiment 1, with the exception of using two platforms, one located in Q1 and the other in Q3 with group A. The two platforms were identical in their dimensions and materials.

\section{Procedure}

The procedure was identical to the procedure of Experiment 1, except where noted.

Pretraining (Days 1-2) The pretraining was identical to the pretraining phase from Experiment 1.

Phase 1 (Days 3-10) All rats received 48 placement trials over eight consecutive days (six trials per day). During these trials, rats were placed directly on the platform, without requiring them to swim to find it, and they remained on the platform for $30 \mathrm{~s}$ before being removed from the pool. As shown in the bottom section of Fig. 1, for group A two platforms were hidden in two different locations, equidistant to landmarks A and $\mathrm{B}$, in the quadrant closest to the landmark and in the opposite quadrant. Rats were placed on each platform the same number of times counterbalanced. A pseudorandom sequence was created for each day with the only restriction that a position (close or far from the landmarks) could not be repeated more than twice in a row (e.g., CFFCFC, FFCCFC, for first and second training days). For group $\mathrm{R}$ a single platform was hidden in the quadrant closer to the landmarks. A probe trial of free swimming was conducted at the end of every other day of training. Probe trials were identical to the ones conducted in Experiment 1.

Phase 2 (Days 11-18) The second phase for groups R and A was identical to the one received by the corresponding groups in Experiment 1, with the only exception being that group A had two platforms available on each trial.

Phase 3 (Days 19-24) Phase 3 was identical to phase 3 conducted in Experiment 1.

\section{Results and discussion}

\section{Latency}

Phase 2 As in Experiment 1, we combined the data into two session blocks, as shown in Fig. 4. There was a large effect of whether or not the rats were searching for one platform, opposite to where it had been in the prior phase, or searching for two platforms. As might be expected, rats were significantly faster at locating a platform when two platforms were available (group Acquisition) compared to a group for which they were searching for a new platform location (group Reversal). This description was confirmed by a Group $\times$ Block ANOVA, which revealed effects of Block $F(3,42)=5.05, p=.004, \eta^{2}{ }_{p}=$ $.27,95 \%$ CI $[.04, .42]$, and Group, $F(1,14)=7.26, p=.017$, $\eta_{p}^{2}=.34,95 \%$ CI $[.01, .59]$, and no interaction, $F<1$.

Rats in group A showed no bias in locating one platform over the other. On each two-session block, the number of times that the $\mathrm{AB}$ platform was located $(0-12)$ averaged

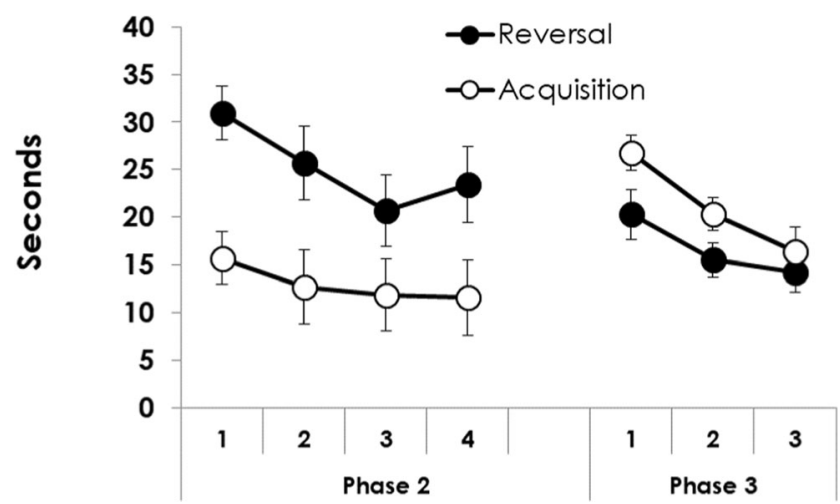

Fig. 4 Latency to reach the platform in each phase of Experiment 2, averaged across two-session blocks. Vertical lines represent the standard error of the mean 
6.14 across the phase and did not vary by block, $F(3,21)=$ $1.87, p=.17$ (note that the analysis based on finding either platform will be the same).

In phase 3 , where only one platform in a new location was available to both groups, the rats that had experienced the reversal found the platform more rapidly than the rats that did not. A Group $\times$ Block ANOVA revealed an effect of Block, $F(2,28)=9.21, p=.001, \eta^{2}=.40,95 \%$ CI [.1, .57], and effect of Group, $F(1,14)=4.76, p=.047, \eta_{p}^{2}=$ $.25,95 \%$ CI $[.000006, .52]$, and no interaction, $F<1$.

\section{Probe trials}

Phase 1 The probe trials were averaged into training halves and analyzed as in Experiment 1. The data are shown in Fig. 5. In phase 1 a Group $\times$ Quadrant $\times$ Block ANOVA revealed a Quadrant $\times$ Block interaction, $F(3,42)=58.82, p<.0001, \eta_{p}^{2}$ $=.81,95 \% \mathrm{CI}[.67, .85]$, with no other effects whose interpretation is not superseded by the interaction, $p \mathrm{~s}>.31$. There were effects of Quadrant in both halves of training, $F_{\mathrm{S}}(3,45) \geq 16.91, p<.0001, \eta^{2} \geq .57,95 \%$ CI $[.29, .66]$.

In the first block of training rats initially preferred the Q4 AC quadrant over all others, $F \mathrm{~s}(1,15) \geq 14.9, p \mathrm{~s} \leq .002, \eta_{p}^{2} \geq$ $.50,95 \%$ CI $[.11, .69]$. The least preferred quadrant was Q2 AC-opposite, differing from the others, $F \mathrm{~s}(1,15) \geq 12.91, p \mathrm{~s} \leq$ $.003, \eta^{2} \geq .46,95 \%$ CI $[.08, .67]$. The AB and AB-opposite quadrants did not differ, $F<1$.

In the second half of the training all rats came to prefer the $\mathrm{AB}$ quadrant more than the others, $F_{\mathrm{s}}(1,15) \geq 8.96, p \mathrm{~s} \leq .009$, $\eta_{p}^{2} \geq .37,95 \%$ CI $[.03, .61]$. Rats also preferred the AC opposite quadrant over the $\mathrm{AC}$ and $\mathrm{AB}$-opposite quadrants,
$F_{\mathrm{s}}(1,15) \geq 71.75, p \mathrm{~s}<.0001, \eta^{2} \geq .87,95 \%$ CI $[.59, .90]$. The $\mathrm{AC}$ and $\mathrm{AB}$-opposite quadrants did not differ, $F<1$.

Phase 2 The same analysis of phase 2, again revealed equivalent swimming patterns between the groups. There was only an effect of Quadrant, $F(3,42)=9.68, p=.00006, \eta^{2}{ }_{p}=.40$, $95 \%$ CI $[.14, .55]$. There were no interactions with Group, $F(3,42)=1.18, p=.35$, Block, $F(3,42) 1.19, p=.32$, or Block $\times$ Group, $F(3,42)=1.55, p=.22$.

The main effect of quadrant is shown in Fig. 5 above "Both Groups Means." The quadrant effect within each group is shown next to each group's data above "Mean," with both groups clearly showing the same general pattern reflected in the main effects. Q3 was the target quadrant in phase 2 for group $\mathrm{R}$ and a quadrant that held one of two available platforms for group A. Time spent swimming in Q3 was greater than time spent in the other three quadrants, $F_{\mathrm{s}}(1,15)>=7.72$, $p \mathrm{~s} \leq .01, \eta_{p}^{2} \geq .34,95 \%$ CI $[.01, .59]$. Swimming in the other quadrants did not differ, $F_{s}<1$.

Phase 3 In phase 3 there was a Group $\times$ Quadrant $\times$ Block interaction, $F(3,42)=5.19, p=.004, \eta^{2}{ }_{p}=.27,95 \%$ CI $[.04$, .43 , that superseded all other effects. In the first half of the training there was a Quadrant $\times$ Group interaction, $F(3,42)=$ 4.66. In the second half of training there was only an effect of Quadrant, $F(3,42)=23.93$, but no interaction, $F<1$.

In the first half of training, rats in group $\mathrm{R}$ preferred to swim in the target Q4 AC quadrant over the other three, $F \mathrm{~s}(1,7) \geq 5.86, p \mathrm{~s} \leq .0496, \eta_{p}^{2} \geq .46,95 \%$ CI $[.00001, .71]$. There were no differences among the non-target quadrants, $F \mathrm{~s}$ $\leq 1.12, p \mathrm{~s} \geq .33$.

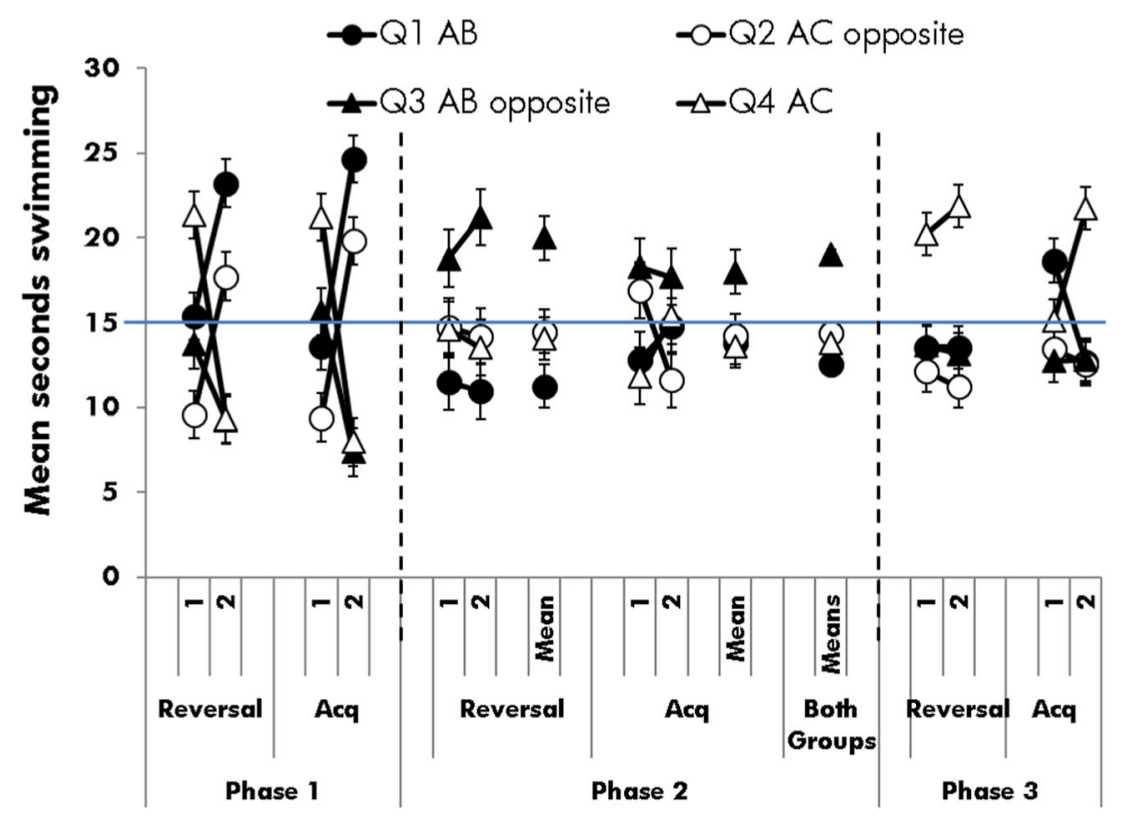

Fig. 5 Mean time spent in each quadrant during the probe trials in each half of each phase of training and testing in Experiment 2. The horizontal line at $15 \mathrm{~s}$ indicates chance performance. Vertical lines on points show the standard error of the mean with between-subjects differences removed to facilitate between-quadrant comparisons within groups 
In the first half of training in group $\mathrm{A}$, the rats tended to prefer the Q1 AB quadrant that they had favored in phase 1, preferring it over Q2 and Q3, $F \mathrm{~s}(1,7) \geq 15.53, p \mathrm{~s} \leq .005 \eta_{p}^{2} \geq$ $.69,95 \%$ CI $[.11, .83]$, with a marginal preference over the target Q4 AC quadrant, $F(1,7)=4.32, p=.076$. There were no other differences among any of the quadrants, $F_{\mathrm{S}}(1,7) \leq 1.63$, $p \mathrm{~s} \geq .24$.

In the second half of training both groups showed the same behavior, favoring the target AC-Q4 quadrant over the other three, $F \mathrm{~s}(1,7) \geq 15.04, p \mathrm{~s} \leq .006, \eta_{p}^{2} \geq .68,95 \%$ CI $[.10, .82]$. There were no other meaningful differences among the quadrants, $F_{\mathrm{S}}(1,7) \leq 1.63, p \mathrm{~s} \geq .24$.

The design of this experiment was arranged so that all rats had the experience of the platform being hidden in two different positions during the initial training, though only group $\mathrm{R}$ received the interference treatment. As in Experiment 1, this difference turned out to be essential, as learning about the new position of the platform during the final phase proceeded faster after the interference treatment.

Exposure to the two platforms in group A may have produced some ambiguity, something that has been claimed to affect context specificity of the information by itself (Rosas et al., 2006; see also Callejas-Aguilera \& Rosas, 2010). However, as shown in Nelson and Callejas-Aguilera (2007), the effect of interference on learning surpassed any potential effect that ambiguity alone might have had. There were no controls in this experiment to test the potential effects of simple ambiguity upon new learning, though a cross-experiment comparison with test performance of control groups in Experiment 1 suggests that ambiguity alone had little effect, if any, upon acquisition of new learning. Alternatively, interference seems to produce a clear improvement in performance when rats are exposed to a new situation in which they have to search for the platform in a new position with respect to a new cue.

\section{General discussion}

Two experiments conducted in the Morris water maze found that prior exposure to an interference treatment could facilitate subsequent acquisition of new spatial learning. In Experiment 1 , reversal training facilitated learning a new platform position with respect to simple acquisition, regardless of the length of acquisition. In Experiment 2, reversal training facilitated learning about the new position of the platform compared to a group that had similar experience with the platform being in different positions of the pool, but that did not have the experience of interference. These results suggest that the reversal treatment facilitates subsequent spatial learning within the water maze because of the interference the reversal produces.

These results agree with the notion that subjects' attention is boosted when the situation becomes ambiguous. In other words, when the prediction error increases because the learning situation has changed, attention is increased. This increase in attention has been claimed to affect stimulus (Kaye \& Pearce, 1984), and context processing (Bouton, 1993; Rosas \& Callejas-Aguilera, 2007). More recently, Alcalá et al. (2019) have claimed that this boost in attention may affect new learning that is acquired after interference has taken place. The present results agree with this latter idea that interference boosts attention generally, affecting learning that takes place after interference (e.g., Easdale et al., 2019; González et al., 2019; Nelson et al., 2018; Shanab \& Cotton, 1970).

However, facilitation of learning after the interference experience may not be a ubiquitous effect. As noted above, Alcalá et al. (2018) failed to find facilitation of learning about a new CS during the interference experience, while finding facilitation of context conditioning. This null result suggests that there could be limitations to the effects of experiencing interference on subsequent learning. In the current experiments, acquisition of new learning was tested in the presence of familiar cues (A and B) and new cue (C). The new cue was essential to resolve the new learning conditions. In that sense, attention towards new stimuli may already be at an asymptote (Larrauri \& Schmajuk, 2008), while attention to a familiar, irrelevant, context should be low and subject to increases with interference. Such an idea is consistent with the differential effects of the interference experience on new learning reported by Alcalá et al. (2018). However, contrary to the experimental design used by Alcalá et al., (2018) in which animals only needed to learn to that the new cue was related to the administration of food, in the current experiments attention to the new cue was not enough to solve the spatial problem. Solution of the spatial problem at testing required attending to the combination of the new cue $\mathrm{C}$, and the familiar cue $\mathrm{A}$. The results of these experiments are better explained if the experience of interference maintained or increased attention to the familiar cues (e.g., Pearce \& Hall, 1980). Although the experimental designs used in these experiments do not allow us to determine whether the experience of interference differentially affects attention to new and familiar cues, some reports in the literature suggest that experiencing interference seems to restore associability of previously pre-exposure cues (e.g., Hall \& Pearce, 1982; see also González et al., 2019). Accordingly, it seems reasonable to suggest that pre-asymptotic attention to both discrete stimuli and contexts should be similarly boosted by interference, as well as to any other source of information present within the learning situation.

It is worth mentioning that the animals did not necessarily need cue $\mathrm{C}$ to locate the platform. The Reversal animals were able to locate the platform when it moved to a new position using only the original A-B cues. Thus, they could have used those cues to learn the new platform location in phase 3 . Nevertheless, it is likely that cue $\mathrm{C}$ was used. In the reversal groups the platform was moved from near the cues to far from 
the cues, but was located on a vector between the cues in both cases (see also Chamizo et al., 2012). Introduction of the new cue $\mathrm{C}$ allows the animals to continue to locate the platform between cues where simply searching between the $\mathrm{AB}$ cues would produce inaccuracies and take more time to locate the platform. The data indicate that they learned phase 3 at least as fast as phase 2 , suggesting that the new cue, which controlled no opposing tendencies, was used.

Alternatively, the experience of interference could have attenuated the difficulties in new learning produced by the initial acquisition training in the control groups, rather than facilitate new learning in the reversal condition. In Experiment 1 , group $\mathrm{AL}$ persisted in swimming in the $\mathrm{AB}$ quadrant during the first half of the test, clearly interfering with either the acquisition or expression of learning the new target location. But, in group AS, which had the same amount of training in the $A B$ quadrant as group $\mathrm{R}$, no such persistence was observed. Thus, it is unlikely that interference training in group $\mathrm{R}$ simply undid the effect of interference that the initial phase 1 training could produce in phase 3 .

A relevant issue in the interpretation of the results of these experiments relates to the length of the interference phase. It is assumed that it is the increase in the prediction error produced by the interference that facilitates learning by an activation of the exploratory mechanism of attention (Le Pelley et al. 2016). However, the interference phase was conducted until both groups reached similar performance and thus, by the end of the interference phase, the local prediction error should be essentially the same in all the groups, regardless of whether they experienced interference or not. Note that this situation is akin to the one that it is usually observed in renewal studies, where context-specificity of extinction is often reported once extinction has been conducted up to the asymptote (for a review, see Bouton, 1993). This result is consistent with the idea discussed above that the interference experience may keep attention to familiar cues high (Pearce \& Hall, 1980). Alternatively, the change in the prediction error could produce something similar to a learning-to-learn effect facilitating the adaptation to new learning settings once the interference is experienced (e.g., Balea, Sanjuan, \& Nelson, 2018; Brow \& Kane, 1988; Harlow, 1949). Thus, as appealing as the interpretation in terms of a general increase of attention as a consequence of the experience with an increase in the prediction error during interference may be, the mechanism of that facilitation is still under discussion, and will need further exploration.

Finally, we believe that the pattern of swimming in group A during Experiment 2 deserves discussion. Group A changed their quadrant preference between phase 1 and phase 2 . In the first phase they spent more time swimming close to the landmarks (Q1), and during the second phase rats spent more time in the opposite quadrant (Q3). Thus, rats changed their preference from the quadrant close to the landmark to the quadrant far from the landmark. However, in both phases the platform was available in both quadrants. The only difference between phase 1 and phase 2, for these animals, is the kind of training received. In phase 1 the rats were placed on each platform, thus the cues were passively associated with the platform as no action was required of the rat. In the phase 1 probe trials, the rats tended to prefer the quadrants near the landmarks, perhaps reflecting some type of effect of physical contiguity (e.g., Christie, 1996; Nelson, Navarro, \& del Carmen Sanjuan, 2019; Rescorla \& Cunningham, 1979) when associating the cues with the platform. But, on those probe trials the animal was active, and the platform was absent, making those trials effective extinction trials for swimming toward the cues. Thus, in phase 2 when swimming was required, the prior non-reinforcement for swimming near the cues may have better primed the rats to avoid those cues and more readily encounter the platform in the opposite quadrant. The tendency of swimming toward the cues was effectively punished in phase 1 probe trials, while swimming away from the cues was effectively rewarded in phase 2 training trials. Despite this change in the rat's preference, group Acquisition learned according their contingency and then showed preference for one of the target quadrants.

At any rate, the results of these experiments suggest that the experience of interference facilitates learning about location cues in a water maze in rats. This result extends previous results reported in rats' Pavlovian (e.g., Alcalá et al., 2019) operant appetitive conditioning (Liberman, 1951; Shanab \& Cotton, 1970) and in human learning (Easdale et al., 2019; González et al., 2019; Nelson et al., 2018) to an aversively motivated spatial learning situation, suggesting that the facilitating effect of experiencing interference on new learning is a general effect found across different species and procedures.

Acknowledgements Research was made possible by Grants PSI201452263-C2-1-P and PSI2014-52263-C2-2-P from the Spanish Ministry of Science and Competitiveness, and Grant R6/6/2014 of the Research Support Plan of the Universidad de Jaén sponsored by the Caja Rural de Jaén, and by the Centre of Animal Production and Experimentation of the Universidad de Jaén. Final preparation of the published material was supported by Grants PGC2018-097769-B-C21 and PGC2018-097769-B-C21 and -C22 from the Spanish Ministry of Science, Innovation, and Universities. Participation of J.A. Alcalá was funded by Grant FPU13/ 03761 from the Ministry of Education, Culture and Sport from Spain. This research was part of J.A. Alcalá's thesis dissertation. J.A. Alcalá is currently a postdoctoral fellow at the University of Leicester. We thank María Victoria Martinez Lorente for her assistance in running part of the experiments.

\section{Compliance with ethical standards}

Conflict of interest None declared.

\section{References}

Alcalá, J.A., Callejas-Aguilera, J.E., Lamoureux, J.A., \& Rosas, J.M. (2019). Discrimination reversal facilitates subsequent acquisition of temporal discriminations in rats' appetitive conditioning (in 
press). Journal of Experimental Psychology: Animal Learning and Cognition. https://doi.org/10.1037/xan0000216

Alcalá, J.A., González, G., Aristizabal, J.A., Callejas-Aguilera, J.E., \& Rosas, J.M. (2018). Discrimination reversal facilitates contextual conditioning in rats' appetitive conditioning. Psicológica, 39, 6487. https://doi.org/10.2478/psicolj-2018-0004

Balea, P., Sanjuan, M. C., \& Nelson, J. B. (2018). Learning to learn in conditioning and extinction in humans. Behavioural Processes, 157 , 148-160. https://doi.org/10.1016/j.beproc.2018.09.005

Beesley, T., Nguyen, K. P., Pearson, D., \& Le Pelley, M. E. (2015). Uncertainty and predictiveness determine attention to cues during human associative learning. The Quarterly Journal of Experimental Psychology, 68, 2175-2199. https://doi.org/10.1080/17470218. 2015.1009919

Bouton, M. E. (1993). Context, time, and memory retrieval in the interference paradigm of Pavlovian learning. Psychological Bulletin, 114, 80-99. https://doi.org/10.1037/0033-2909.114.1.80

Bouton, M. E. (1997). Signals for whether versus when an event will occur. In M. E. Bouton \& M. S. Fanselow (Eds.), Learning, motivation, and cognition: The functional behaviorism of Robert $C$. Bolles (pp. 385-409). Washington, DC: American Psychological Association.

Brow, A.L., \& Kane, M. J. (1988). Preschool children can learn to transfer: Learning to learn and learning from example. Cognitive Psychology, 20, 493-523. https://doi.org/10.1016/0010-0285(88) 90014-X

Callejas-Aguilera, J. E., \& Rosas, J. M. (2010). Ambiguity and context processing in human predictive learning. Journal of Experimental Psychology: Animal Behavior Processes, 36, 482-494. https://doi. org/10.1037/a0018527

Chamizo, V. D. (2003). Acquisition of knowledge about spatial location: Assessing the generality of the mechanism of learning. Quarterly Journal of Experimental Psychology, 56B, 102-113. https://doi.org/ 10.1080/02724990244000205

Chamizo, V. D., Rodríguez, C. A., Espinet, A., \& Mackintosh, N. J. (2012). Generalization decrement and not overshadowing by associative competition among pairs of landmarks in a navigation task. Journal of Experimental Psychology. Animal Behavior Processes, 38, 255-65. https://doi.org/10.1037/a0029015

Christie, J. (1996). Spatial contiguity facilitates Pavlovian conditioning. Psychonomic Bulletin \& Review, 3, 357-359. https://doi.org/10. 3758/BF03210760

Courville, A. C., Daw, N. D., \& Touretzky, D. S. (2006). Bayesian theories of conditioning in a changing world. Trends in Cognitive Sciences, 10, 294-300. https://doi.org/10.1016/j.tics.2006.05.004.

Easdale, L. C., Le Pelley, M. E., \& Beesley, T. (2019). The onset of uncertainty facilitates the learning of new associations by increasing attention to cues. Quarterly Journal of Experimental Psychology, 72, 193 -208. https://doi.org/10.1080/17470218.2017.1363257

González, G., Alcalá, J.A., Callejas-Aguilera, J. E., \& Rosas, J. M. (2019). Experiencing extinction with a non-target cue facilitates reversal of a target conditioned inhibitor in human predictive learning. (in press) Behavioural Processes. https://doi.org/10.1016/j.beproc. 2019.103898

Hall, G., \& Pearce, J. M. (1982). Restoring the associability of a preexposed CS by a surprising event. The Quarterly Journal of Experimental Psychology Section B: Comparative and Physiological Psychology, 34, 127-140. https://doi.org/10.1080/ 14640748208400881

Harlow, H. F. (1949). The formation of learning sets. Psychological Review, 56, 51-65. https://doi.org/10.1037/h0062474

Horne, M. R., Gilroy, K. E., Cuell, S. F., \& Pearce, J. M. (2012). Latent spatial learning in an environment with a distinctive shape. Journal of Experimental Psychology. Animal Behavior Processes, 38, 13947. https://doi.org/10.1037/a0027288
Jeffery, K. J. (2010). Theoretical accounts of spatial learning: A neurobiological view (commentary on Pearce, 2009). Quarterly Journal of Experimental Psychology, 63, 1683-99. https://doi.org/10.1080/ 17470210903540771

Kaye, H., \& Pearce, J. M. (1984). The strength of the orienting response during Pavlovian conditioning. Journal of Experimental Psychology. Animal Behavior Processes, 10, 90-109. https://doi. org/10.1037/0097-7403.10.1.90

Larrauri, J. A, \& Schmajuk, N. A. (2008). Attentional, associative, and configural mechanisms in extinction. Psychological Review, 115, 640-676. https://doi.org/10.1037/0033-295X.115.3.640

Lattal, K. M., Mullen, M. T., \& Abel, T. (2003). Extinction, renewal, and spontaneous recovery of a spatial preference in the water maze. Behavioral Neuroscience, 117, 1017-1028. https://doi.org/10. 1037/0735-7044.117.5.1017

Le Pelley, M. E. (2004). The role of associative history in models of associative learning: A selective review and a hybrid model. Quarterly Journal of Experimental Psychology, 57B, 193-243. https://doi.org/10.1080/02724990344000141

Le Pelley, M. E., Beesley, T., \& Griffiths, O. (2011). Overt attention and predictiveness in human contingency learning. Journal of Experimental Psychology Animal Behavior Processes, 37, 220 229. https://doi.org/10.1037/a0021384

Le Pelley, M.E., Mitchell, C.J., Beesley, T., George, D.N. \& Wills, A. J. (2016). Attention and associative learning in humans: An integrative Review. Psychological Bulletin, 142, 1111-40. https://doi.org/10. 1037/bul0000064

Liberman, A. M. (1951). A comparison of transfer effects during acquisition and extinction of two instrumental responses. Journal of Experimental Psychology, 41, 192. https://doi.org/10.1037/ h0062259

Mackintosh, N.J. (1975). A theory of attention: Variations in the associability of stimuli with reinforcement. Psychological Review, 82, 276-298. https://doi.org/10.1037/h0076778

Morris, R. G. M. (1981). Spatial localization does not require the presence of local cues. Learning \& Motivation, 12, 239-260. https://doi.org/ 10.1016/0023-9690(81)90020-5

Nelson, J.B. (2016). A robust function to return the cumulative density of non-central F distributions in Microsoft Office Excel. Psicológica, 37, 61-83. Retrieved from https://www.redalyc.org/articulo.oa?id= 16943586004

Nelson, J. B., \& Callejas-Aguilera, J. E. (2007). The role of interference produced by conflicting associations in contextual control. Journal of Experimental Psychology: Animal Behavior Processes, 33, 314 326. https://doi.org/10.1037/0097-7403.33.3.314

Nelson, J.B., Fabiano, A.M., \& Lamoureux, J.A. (2018). The effects of extinction-aroused attention on context conditioning. Learning \& Memory, 25, 165-175. https://doi.org/10.1101/lm.046201.117

Nelson, J. B., Navarro, A., \& del Carmen Sanjuan, M. (2019). Conditioned anticipatory outcome searching in humans. Behavioural processes, 164, 237-251. https://doi.org/10.1016/j. beproc.2019.05.015

Pearce, J. M. (2009). The 36th Sir Frederick Bartlett Lecture: An associative analysis of spatial learning. Quarterly Journal of Experimental Psychology, 1-20. https://doi.org/10.1080/ 17470210902805589

Pearce, J.M. \& Hall, G. (1980). A model for Pavlovian learning: Variations in the effectiveness of conditioned but not of unconditioned stimuli. Psychological Review, 87, 532-552. https://doi.org/ 10.1037/0033-295X.87.6.532

Prados, J., Chamizo, V. D., \& Mackintosh, N.J. (1999). Latent inhibition and perceptual learning in swimming pool navigation task. Journal of Experimental Psychology: Animal Behavior Processes, 25, 37 44. https://doi.org/10.1037/0097-7403.25.1.37 
Prados, J., Manteiga, R. D., \& Sansa, J. (2003). Recovery effects after extinction in the Morris swimming pool navigation task. Learning \& Behavior, 31, 299-304. https://doi.org/10.3758/BF03195991

Prados, J., Sansa, J., \& Artigas, A. (2008). Partial reinforcement effects on learning and extinction of place preferences in the water maze. Learning \& Behavior, 36, 311-8. https://doi.org/10.3758/LB.36.4. 311

Rescorla, R. A., \& Cunningham, C. L. (1979). Spatial contiguity facilitates Pavlovian second-order conditioning. Journal of Experimental Psychology: Animal Behavior Processes, 5(2), 152-161. https://doi. org/10.1037/0097-7403.5.2.152

Roberts, A. D., \& Pearce, J. M. (1999). Blocking in the Morris swimming pool. Journal of Experimental Psychology. Animal Behavior Processes, 25, 225-35. https://doi.org/10.1037/0097-7403.25.2.225

Rosas, J. M., \& Callejas-Aguilera, J. E. (2007). Acquisition of a conditioned taste aversion becomes context dependent when it is learned after extinction. Quarterly Journal of Experimental Psychology, 60, 9-15. https://doi.org/10.1080/17470210600971519.

Rosas, J.M., Callejas-Aguilera, J.E, Ramos-Álvarez, M.M., \& Abad, M.J. (2006). Revision of Retrieval Theory of Forgetting: What does make information context-specific? International Journal of Psychology and Psychological Therapy, 6, 147-166. Retrieved from https:// www.ijpsy.com/volumen6/num2/136.html

Schmajuk, N. A., Lam, Y. W., \& Gray, J. A. (1996). Latent inhibition: A neural network approach. Journal of Experimental Psychology: Animal Behavior Processes, 22, 321-349. https://doi.org/10.1037/ 0097-7403.22.3.321

Shanab, M. E., \& Cotton, J. W. (1970). Effects of runway training on behavior in the T-maze. Psychonomic Science, 19, 129-130. Retrieved from https://link.springer.com/content/pdf/10.3758\% 2FBF03335510.pdf

Sutherland, R. J., \& Linggard, R. C. (1982). Being there: A novel demonstration of latent spatial learning in the rat. Behavioral and Neural Biology, 36, 103-107. https://doi.org/10.1016/S0163-1047(82) 90101-7

Publisher's note Springer Nature remains neutral with regard to jurisdictional claims in published maps and institutional affiliations. 